\title{
Société Suisse de Gastro-Entérologie
}

Assemblée annuelle, les 29 et 30 octobre 1965 à Geneve

Schweizerische Gesellschaft für Gastroenterologie

Jahresversammlung in Genf, 29. und 30. Oktober 1965

Communications - Vorträge Fin - Schluss

Halter, F.; Ganguli, P. C; Marshall, M. and Crean, G. P. (Edinburgh):

Preliminary Communication of the Effect of Gastrin II on Acid Secre

tion in Conscious Rats with Chronic Gastric Fistula 194

Hotz,H. W. und A ufdermaur, M. (Luzern): Postvitale Magenschleimhautver-

änderungen 202

Kistler, H. J.; Schmid, J. R.; Pfennínger, E.; Haemmerli, U. P. und Fríck,

P. G. (Zurich): Untersuchungen über die intestinale Absorption von

H3-Folsäure bei idiopathischer Sprue 204

Antoníolí, J. A.; Robinson, J. W. L.; Fasel, J. et Vannottí, A. (Lausanne):

Effet du méthotrexate in vivo sur Гintestin grêle du rat 217

Saegesser, F. et Waridel, D. (Lausanne): Le risque de cancérisation du gros

intestin dans la rectocolite ulcéro-hémorragique chronique 225

Mégevand, R. (Geneve): Hémopéritoine par hépatome sous-capsulaire rompu, sectoriectomie et guérison 238

Resume du protocole de $\Gamma$ assemblée administrative du 29 novembre 1965245

13 Gastroenterologia, Vol. 106, No. 4 (1966) 